\title{
Modified Predictive Control for a Class of Electro-Hydraulic Actuator
}

\author{
Abdulrahman A.A. Emhemed*, Rosbi Bin Mamat*, Ahmad ‘Athif Mohd Faudzi**, \\ Mohd Ridzuan Johary**, Khairuddin Osman*** \\ * Department of Control and Mechatronic Engineering, Universiti Teknologi Malaysia, 81300 Skudai, Malaysia \\ ** Centre for Artificial Intelligence and Robotics (CAIRO), Universiti Teknologi Malaysia, 81300 Skudai, Malaysia \\ *** Department of Industrial Electronics, Universiti Teknikal Malaysia Melaka, 76100 Durian Tunggal, Malaysia
}

\section{Article Info}

Article history:

Received Aug 23, 2015

Revised Dec 28, 2015

Accepted Dec 12, 2016

\section{Keyword:}

Modified predictive control Electro-hydraulic actuator Position control

\begin{abstract}
Many model predictive control (MPC) algorithms have been proposed in the literature depending on the conditionality of the system matrix and the tuning control parameters. A modified predictive control method is proposed in this paper. The modified predictive method is based on the control matrix formulation combined with optimized move suppression coefficient. Poor dynamics and high nonlinearities are parts of the difficulties in the control of the Electro-Hydraulic Actuator (EHA) functions, which make the proposed matrix an attractive solution. The developed controller is designed based on simulation model of a position control EHA to reduce the overshoot of the system and to achieve better and smoother tracking. The performance of the designed controller achieved quick response and accurate behavior of the tracking compared to the previous study.
\end{abstract}

Copyright (C) 2016 Institute of Advanced Engineering and Science. All rights reserved.

\section{Corresponding Author:}

Abdulrahman A.A. Emhemed, Department of Control and Mechatronic Engineering, Faculty of Electrical Engineering, Universiti Teknologi Malaysia, 81300 Skudai, Malaysia.

Email: abdo_83f@yahoo.com

\section{INTRODUCTION}

Generalized Predictive Control (GPC) is considered one of the most popular Model Predictive Control (MPC) algorithms in industry. GPC with integral action is derived based on the minimization of a modified predictive performance criterion. An important advantage of this type of predictive control is its ability to cope with hard constraints on controls and states. Recently, several predictive control approaches where proposed to solve EHA Actuators problems. EHA position control systems are very important for the industrial application of control systems, e.g., aircraft flight control, remote robot position control, due to their characteristics of fast response, accurate positioning, and so on. Electro-Hydraulicsystems are complex, nonlinear and difficult to identify and control. A close investigation has suggested that the problems are mostly related to the nature of hydraulic functions. First of all, in EHA, flexible connecting hoses, large volume of fluid under compression and trapped air in the hydraulic fluid lead to high compliance. This paper's main contribution is modified predictive control designed to enhance position control issue for EHA plant. The proposed method is using a new matrix combined with optimal tuned move suppression factor. The GPC method designed and implemented to overcome various control problems in one algorithm [1]. They are capable to stabilize processes with variable parameters. It can also adapt with model order which changes immediately provided that the input/output data are valuable to allow well-founded plant identification. Bigdeli and Haeri implemented GPC algorithm for Position Control of an Ultrasonic Motor (USM). The motor possess heavy nonlinear, and load dependent characteristics such as dead-zone. These properties have made big challenge for Position and velocity control of the plant. The GPC improved the performance of the motor for both position tracking and disturbance rejection [2]. The presented GPC 
position control scheme provides damping noise, fast response, and good rejection of uncertainties for induction motor. The position loop is regulated with a linear GPC controller and the current loops incorporate the Space Vector Pulse Width Modulation (SVPWM) with PI control [3]. The Investigation has achieved perfect results based on time delay enhancement for hydraulic position control system (HPCS) using modified predictive control technique. Both sensor-to-controller (S-C) and controller-to-actuator (C-A) network-induced delays are modeled as Markov chains [4]. A research group from The University of Manitoba, Canada applied GPC control to a class of position control of hydraulic manipulator. The investigated experimental has been done through data connection between the computer and the real plant through acquisition card. An on-line estimation of time varying plant parameters and identification achieved. The influence of GPC design parameters was also experimentally studied and observed [5]. MPC advantages are detailed in [6], [7]. Designing a predictive controller for integrating processes is a big challenging step because of the number of adjustable parameters (prediction horizon, control horizon, move suppression coefficient, and the sampling time) that affect the closed-loop performance. The novel analytical method has been achieved good performance include (set point tracking, overshoot limitation, and disturbance rejection) based on simulation results for varies processes [8]. The ship dynamic positioning system has some characteristics such as nonlinear, large delay and strong coupling, which is difficult to structure the accurate mathematical model and very complicated to model it. So the fuzzy predictive control used to control the ship dynamic positioning system (SDPS) in three degrees of freedom (DOF) has been investigated and the simulation results show that the fuzzy predictive control can orient the vessels effectively [9]. An improved robust model predictive controller (RMPC) is presented based on model reference adaptive system (MRAS) for three degree freedom satellite. The control designed because of external disturbance is compensated on the stability and performance of closed loop system. Then the results of proposed RMPC compared to generalized incremental predictive control (GIPC) andshow that the RMPC method is more robust than the GIPC method [10]. An Electro-Pneumatic clutch actuator controlled on/off valves using Pulse Width Modulation (PWM) and Nonlinear Model Predictive Control (NMPC) approach is applied to design an explicit reference tracking. The performance of the designed controller has better quality in comparison to an explicit quantized NMPC controller without PWM [11]. The presented article has these outlines:

- Section I: Introduction and background studies of the EHA controlled by predictive control.

- Section II: The experimental setup and identification methods for varies orders of the EHA are presented.

- $\quad$ Section III: The modified predictive control, where the proposed matrix are explained.

- $\quad$ Section IV: The tuning strategy and computations of the control parameters are presented.

- Section V: Simulation design of the proposed controller and comparison with previous study based on model of position control EHA.

- Section VI: Discussion and conclusion presents in results are summarized and discussed in the light of our objectives.

\section{MODEL IDENTIFICATION OF THE EHA}

In EHA system operation, hydraulic actuator causes linear action from different pressure in the cylinder by pushing against the piston. Generally, the actuator consists of two oil chambers, separated by the piston. The result of oil flows moving into and out of the chambers driving the piston and generate the required pressures to move the load of the actuator [12]. A linear type actuation of the EHA system using a single-ended cylinder and controlled with ON/OFF valve is considered in the experimental design. The experimental setup and the identification responses of the EHAshown in figure 1. 

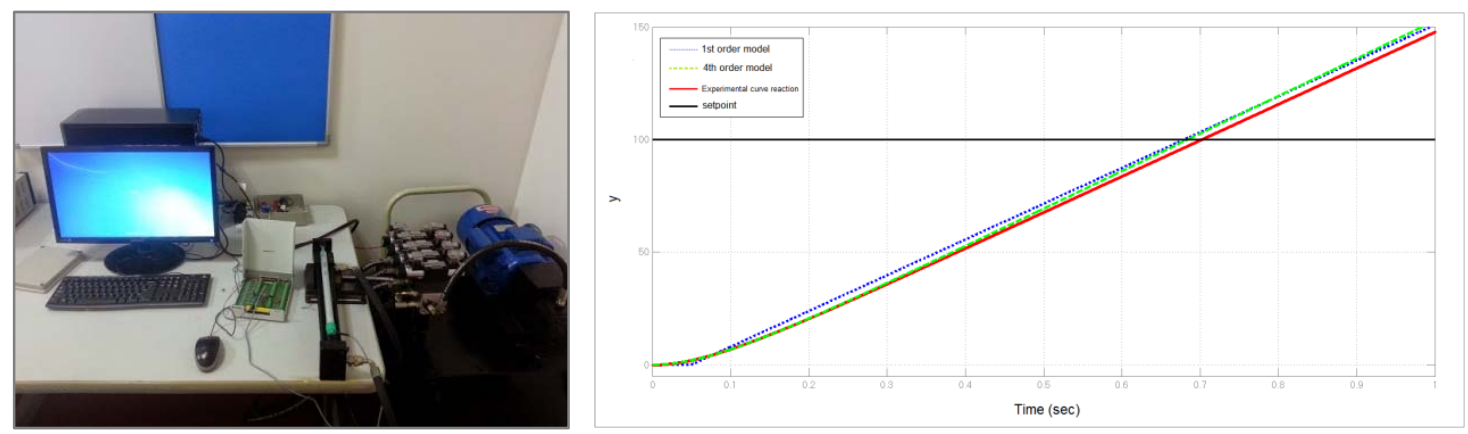

Figure 1. (a) Real experimental setup of the EHA, (b) Identification responses of of the EHA

In the system identification process, previously, this EHA process is identified using ARX identification technique. In this paper the identification described based on curve reaction approximation techniques.The first order step response system identification methods approximated the parameters based on the input/output the model is determined by applying non-parametric system identification based on experimental response for the EHA. The process gain describes based on the steady state effect of the input change to the change of the output. The dead time can be directly read from the output response [13], [14].

$$
G_{1 s t}=\frac{k_{p} e^{-\tau_{d} s}}{s}=\frac{1.587 e^{-0.05 s}}{s}
$$

The first order approximation would be derived as a process gain $k_{p}$, dead time $\tau_{d}$. The approximation from an experimental test of the dynamic system and compared with the identified fourth order system identification in unit step response. Time delay adjusted to be more than zero to achieve a satisfactory match and simplify the control parameters analysis.

$$
G_{4 t h}=-\frac{0.15 s^{3}+5 \times 10^{4} s^{2}+7 \times 10^{6} s-5 \times 10^{9}}{s^{4}+1055 s^{3}+2.5 \times 10^{8} s^{2}+3.4 \times 10^{9} s-0.5}
$$

\section{MODIFIED PREDICTIVE CONTROL}

The following linear, discrete time, single input/single output ARX model representation by (3):

$$
A\left(z^{-1}\right) y(t)=B\left(z^{-1}\right) z^{-d} u(t-1)+e(t)
$$

where $A$ and $B$ are polynomials in the backward shift operator $z^{-1}$ as below:

$$
\begin{aligned}
& A\left(z^{-1}\right)=1+a_{1} z^{-1}+a_{2} z^{-2}+\cdots+a_{n a} z^{-n a} \\
& B\left(z^{-1}\right)=b_{0}+b_{1} z^{-1}+b_{2} z^{-2}+\cdots+b_{n b} z^{-n b}
\end{aligned}
$$

where $n a \geq n b, d$ is time delay, na is number of poles, $n b$ is number of zeros.

The predictive control algorithm consists of applying a control sequence that minimizes a multistage cost function of the form

$$
J\left(M_{\min }, M_{\max }, M_{c h}\right)=\sum_{M_{\min }}^{M_{\max }} \delta(\mathrm{j})[\hat{y}(t+j \mid t)-\psi(t+j)]^{2}+\sum_{j=1}^{M_{c h}} \lambda_{c h}(j)[\Delta u(t+j-1)]^{2}
$$

where $\hat{y}(t+j \mid t)$ is an optimum j-step ahead prediction of the system output on data up to time t. $\psi(t+j)$ is the future reference trajectory, $M_{\min }$ is the minimum costing horizon $M_{\min }=d+1$, the maximum costing horizon is $M_{\max }=d+M_{c h}$, and $M_{c h}$ known as the control horizon , respectively. $\delta(\mathrm{j})$, and $\lambda_{c h}(j)$ are weighting sequences.

Since the control signal that is actually sent to the process is the first element of vector $u$ (receding strategy), it is given by: 


$$
\Delta u=\left(G^{T} G+W_{\lambda}\right)^{-1} G^{T}(\psi-f)
$$

where $G$ is the dynamic matrix, $W_{\lambda}=\lambda I$ where $I$ is diagonal matrix, $\psi$ is a vector of the reference trajectory, and $f$ is a matrix calculated using the Diophantine equation.

In our proposed matrix another strategy effects different type of models such as first, second, and high order systems, and gives more quality responses compared to the previous study especially in terms of overshoot. The proposed matrix depends on the suppression coefficient $\lambda$, the diagonal matrix, and other values created from the open-loop response of the original system.The proposed strategy is implemented to different processes to evaluate the performance of a new strategy approach of the modified predictive method. Let's assume $G$ is the open loop step response sampling data of the system model as:

$$
G=\left[\begin{array}{llllll}
g_{i+1} & g_{i+2} g_{i+3} g_{i+4} & g_{i+5} & \cdots & \cdots & g_{i+N}
\end{array}\right]
$$

where $g_{i+N}$ - step response coefficients.

The modified matrix $\Phi$ within $\mathrm{M}=6$ is:

$$
\begin{aligned}
& W_{\text {pro }}=\frac{\lambda}{x \omega} \times \Phi \\
& \Phi=\left[\begin{array}{cccccc}
1 / 2 \omega & \left(g_{i+2}-g_{i+1}\right)\left(g_{i+3}-g_{i+2}\right) & \left(g_{i+4}-g_{i+3}\right) & \left(g_{i+5}-g_{i+4}\right)\left(g_{i+6}-g_{i+5}\right) \\
\left(g_{i+2}-g_{i+1}\right) & 1 / 2 \omega & g_{i+1} & 0 & g_{i+2} & 0 \\
\left(g_{i+3}-g_{i+2}\right) & g_{i+4} & 1 / 2 \omega & g_{i+2} & 0 & g_{i+3} \\
\left(g_{i+4}-g_{i+3}\right) & 0 & g_{i+3} & 1 / 2 \omega & g_{i+3} & 0 \\
\left(g_{i+5}-g_{i+4}\right) & g_{i+3} & 0 & g_{i+2} & 1 / 2 \omega & g_{i+4} \\
\left(g_{i+6}-g_{i+5}\right) & 0 & g_{i+2} & 0 & g_{i+1} & 1 / 2 \omega
\end{array}\right]
\end{aligned}
$$

\section{TUNING STRATEGY}

Model predictive control family mostly used for industrial processes. The GPC performance objective is very similar to the DMC but is minimized via recursion on the Diophantine identity by Clarke [1], [15]. Nevertheless, GPC reduces to the DMC algorithm polynomial that modified the predicted output trajectory is assumed to be unity; therefore, the DMC tuning strategy can be directly applied to GPC controller [16]. Another study based on Cooper's strategy was implemented for Dual Adaption Generalized Predictive Control (DA-GPC) by [17]. The modified predictive method will have the same prediction horizon equation as proposed by Cooper [18] in terms of $P$ prediction horizon and will be compared with Cooper's method. The tuning procedure for control parameters $P, M$, and $\lambda$ as follow:

- $\quad$ Prediction horizon $P$ : normally chosen based on a rise time of $95 \%$ of the process steady state. A more explicit tuning correlation was proposed by [18] for the selection of $P$ as follows:

$$
P=\operatorname{Int}\left(\frac{5 \tau_{d} \sqrt{10}}{T_{s}}\right)+\operatorname{Int}\left(\frac{\tau_{d}}{T_{s}}+1\right)
$$

- Control horizon $M$ :In the GPC formulation, selecting a value of the control horizon $\mathrm{M}>1$ produces a more robust controller at the cost of increased computation load. Several researchers have proposed to set the value of $(M=1)$ [1],[15],[19]. Rossiter [20] stated $(M \geq 3)$, Abu-Ayyad [21], [22] stated $M \approx$ 5 to 10 . In this paper comparison will be presented with previous study by Cooper provided analytical formula for $\mathrm{M}$ as follows.

$$
M \approx \operatorname{Int}\left(\frac{\tau_{d} \sqrt{10}}{T_{s}}\right)+\operatorname{Int}\left(\frac{\tau_{d}}{T_{s}}+1\right)
$$

In the modified predictive method the control horizon chosen as ( $M \approx 5$ to 10$)$.

- The move suppression coefficient $\lambda$ : several researchers enhanced the predictive control based on proposed tuning methods for tune $\lambda$. First tuning formula suggested by a team from Department of Chemical Engineering, University of Alberta Edmonton, Canada [17] as $\lambda=\lambda_{r e f} \cdot B[1]^{2}$. In addition, the value of $\lambda_{\text {ref }}$ can be calculated based on tuned during operation to improve the overall performance and $B[1]$ is the nominator polynomial. Banerjee and Shah [23] suggested simple robust tuning guidelines for 
a process under Generalized Predictive Control using frequency response technique where the small gain criterion provides useful information about the stability and performance of GPC and the system stability under GPC only if the value of $\lambda$ approximately exceeds 1 . Another researchers proposed to tune the move suppression weight based on the following relationship: $\lambda=m P+\pi$. Where $m, \pi$ are constants [24]. A new technique based on regression model techniques for optimal $\lambda$ suggested by [25], Analytical expression for $\lambda$ based on Cooper's derivation is presented as follows:

$$
\begin{aligned}
& \lambda=f\left[k_{p} \cdot T_{s}\right]^{2} \\
& f=\frac{1}{10 M}\left[\frac{M^{2}(P-k+1)^{3}}{3}-0.08 M^{3}(P-k+1)^{2}\right] \\
& k=\operatorname{int}\left(\operatorname{Int}\left(\frac{\tau_{d}}{T_{s}}+1\right)\right)
\end{aligned}
$$

where $k_{p}$ is process gain, $\tau_{d}$ is time delay, $T_{s}$ is sampling time.

\section{CONTROL DESIGN AND DISCUSSION}

Several issues in predictive control tuning become apparent and firstly can be explained when the strategy is designed and tested on simulation before tested on real time application. These issues related to the set-point tracking, overshoot, and disturbances. Previous investigations have established that a significant advantage of MPC over PID controllers is that PID control must be tuned to reject disturbances to have good control, which is not necessary in many instances when using predictive control strategies [16]. The objective of simulation design is to modified the predictive control and testing the controller before verify it in realtime experiment. This is important when dealing with complicated instrument which is not easy to use and install. The simulation analysis and design helps to reduce the time taken than experimentally in designing, tuning and upgrading the controller.

Based on the position model step response the plant can be approximated by a first-order plus dead time (FOPTD) integrating as the process gain $k_{p}=1.58$ and the time delay estimated as $\tau_{d}=0.05$ to get the value for move suppression. The predictive horizon was $P=20$, the control horizon was $M=5$ for Cooper's method. The move suppression for the Cooper method is $\lambda=0.15$, and for the optimal tuning is $\lambda_{\text {opt }}=0.03$,

The tuning value for the move suppression coefficient $\lambda_{\text {opt }}$ is estimated empirically and the parameter $\mathrm{x}$ estimated as $\mathrm{x}=20$. Best performance achieved with $\lambda_{\text {opt }}=0.03$ where faster reaching to the setpoint that is clear from the yellow lines to simplify the estimation of the tracking. These tuning values with their performances based on rise time and settling time are presented in the figures (2-4) and the comparison analysis of the tuning performance descripes in Figure 5.

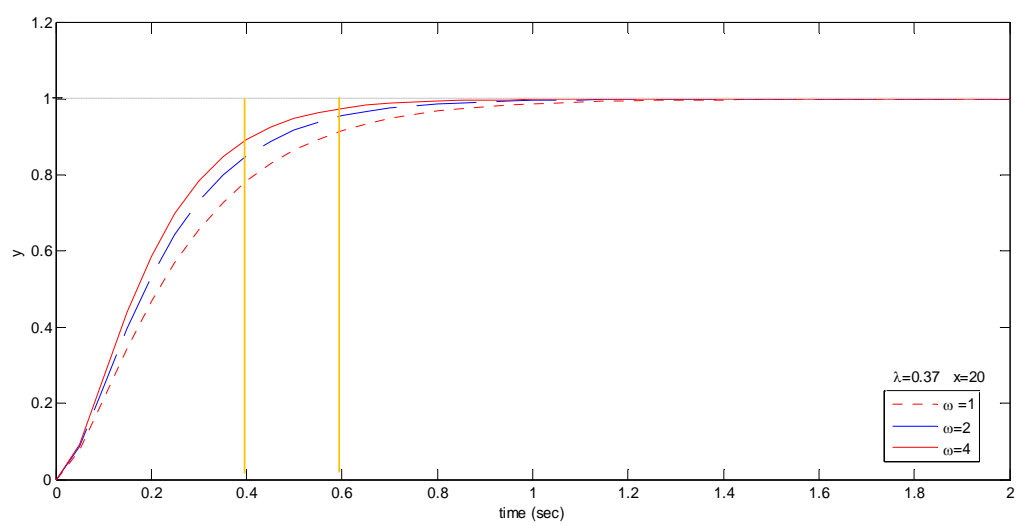

Figure 2. Different values of the parameter tuning $\omega$ based on $\lambda_{\text {opt }}=0.37$ 


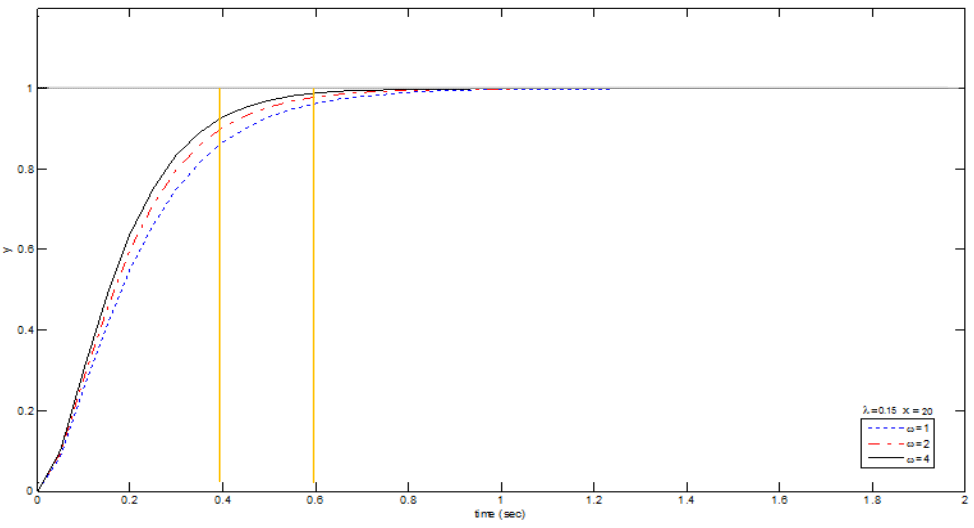

Figure 3. Different values of the parameter tuning $\omega$ based on $\lambda_{\text {opt }}=0.15$

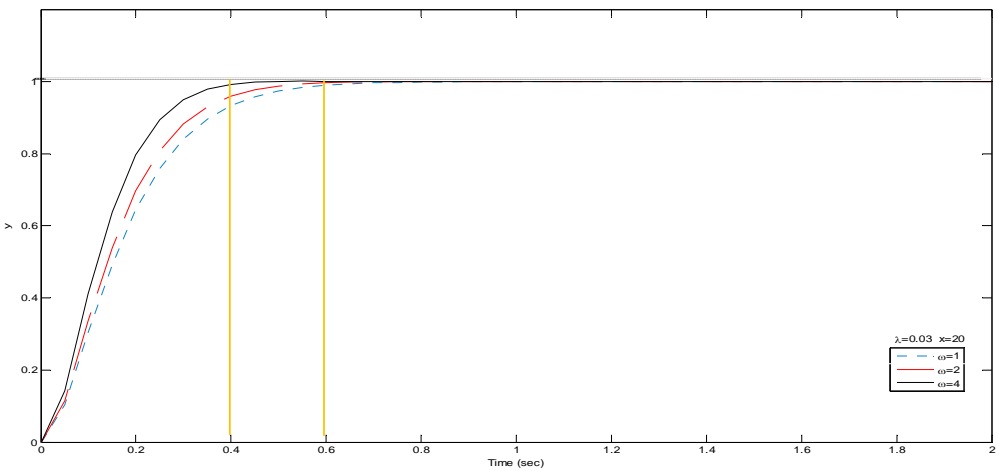

Figure 4. Different values of the parameter tuning $\omega$ based on $\lambda_{\text {opt }}=0.03$

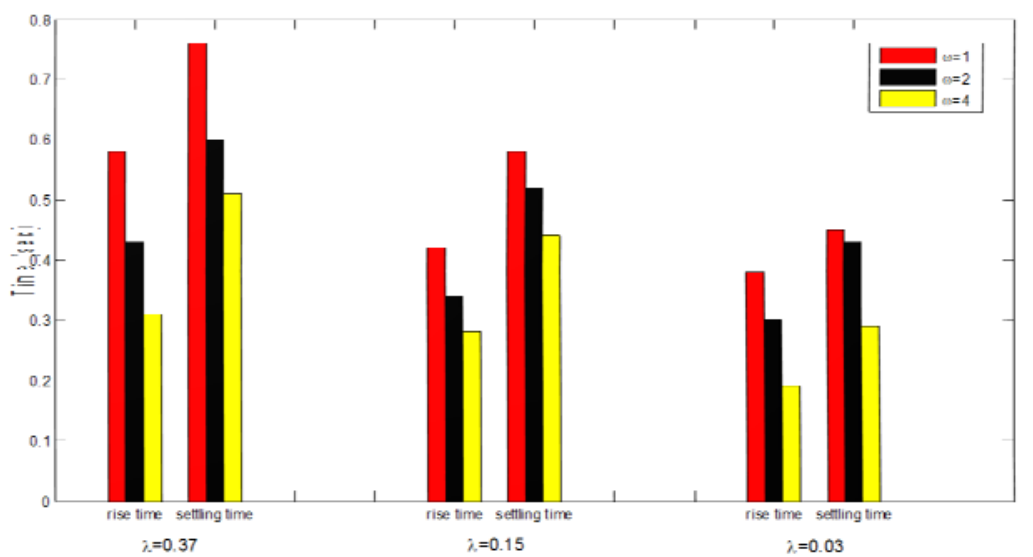

Figure 5. Analysis of different values of the parameter tuning $\omega$ based on different $\lambda_{\text {opt }}$

The analysis testto evaluate the modified predictive method presented with different values of $\lambda_{\text {opt }}$ with varies values of the parameter tuning $\omega$ as shown in Figure 5. Best modified predictive response achieved with $\omega=4$ and $\lambda_{\text {opt }}=0.03$ because of its fast to reach the set point.

The position model results with $\lambda_{o p t}=0.15, \lambda_{o p t}=0.03$ and $\omega=4$ presented in Figure 6 and the performanceanalysis in Table 1 . All the modified predictive responses are demonstrating the shortest settling time, rise time and no overshoot. On theother hand Cooper's method response is unattractive because of its overshoot and slow tracking compare to the modified predictive method. 

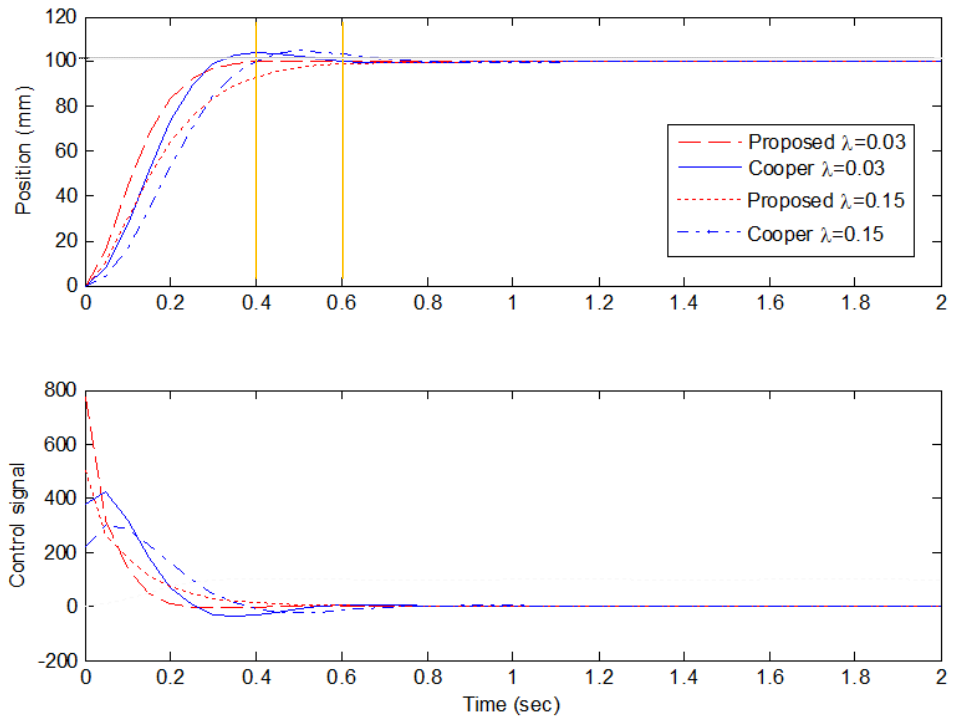

Figure 6. Close loop control responses based on different control schemes with varies $\lambda_{\text {opt }}$ of the EHA system

Table 1. Comparison Results between the Modified Method and Cooper's Method for The EHA System.

\begin{tabular}{ccccc}
\hline $\begin{array}{c}\text { Simulation } \\
\text { performance }\end{array}$ & \multicolumn{2}{c}{$\lambda=0.15$} & \multicolumn{2}{c}{$\lambda=0.03$} \\
Cooper's & mothod & $\begin{array}{c}\text { methied } \\
\text { method }\end{array}$ & $\begin{array}{c}\text { Cooper's } \\
\text { method }\end{array}$ & $\begin{array}{c}\text { modified } \\
\text { method }\end{array}$ \\
\hline Overshoot (\%) & 4.4 & 0 & 4.5 & 0 \\
Rise time (sec) & 0.30 & 0.29 & 0.22 & 0.19 \\
Settling time(sec) & 0.59 & 0.43 & 0.40 & 0.29 \\
\hline
\end{tabular}

\section{CONCLUSION}

The modified predictive control is chosen as representative model predictive control technique and is applied to simulation studies of position control model of EHA. The methodology of analysis considers a dimensionless parameter in order to get general results which could be exercised to any specific real case. The control performance of the modified controller is compared with Cooper's method using fast reacting process. The closed-loop responses are compared and the differences and similarities are explained on the basis of the structure of the control schemes. The simulation tests for the control parameters in some tuning cases for the model EHA position control are given to verify the effectiveness of the modified predictive control. The modified method does not have overshoot, fast to reach the set point, and smoother than Cooper's method.

\section{REFERENCES}

[1] Clarke DW, Mohtadi C, Tuffs PS. Generalized predictive control—Part I. The basic algorithm. Automatica. 1987; 23(2): 137-148.

[2] Bigdeli N, Haeri M. Position control of an ultrasonic motor using generalized predictive control. In Control, Automation, Robotics and Vision Conference (ICARCV). 2004; 1957-1962.

[3] Egiguren PA, Caramazana OB, Etxeberria JAC. Linear generalized predictive position control of induction motor drives. In IECON 2011-37th Annual Conference on IEEE Industrial Electronics Society. 2011; 1922-1927.

[4] Yu B, Shi Y. GPC-based remote control for hydraulic position control systems in a networked environment. In American Control Conference. 2009; 1: 5558-5563.

[5] Sepehri N, Wu G. Experimental evaluation of generalized predictive control applied to a hydraulic actuator. Robotica. 1998; 16(4): 463-474.

[6] Camacho EF, Bordons C. Model Predictive Control. Springer London. 2004.

[7] Maciejowski JM. Predictive Control: With Constraints. Prentice Hall-Technology \& Engineering. 2002.

[8] Danielle D, Cooper DJ. Tuning guidelines of a dynamic matrix controller for integrating (non-self-regulating) processes. Industrial \& engineering chemistry research.2003; 42(8): 1739-1752.

[9] Pirouzmand F. Robust model predictive control based on MRAS for satellite attitude control system. International Journal of Electrical and Computer Engineering (IJECE). 2014; 4(1):81-92.

[10] Liyun W, Jianmei X, Xihuai W. Ship dynamic positioning systems based on fuzzy predictive control. TELKOMNIKA Indonesian Journal of Electrical Engineering. 2013; 11(11): 6769-6779. 
[11] Grancharova A. Explicit MPC of Constrained Nonlinear Systems with Quantized Inputs.Lecture Notes in Control and Information Sciences. 2009; 384: 371-380.

[12] Ghazali R, Sam Y, Rahmat M, Has Z. Adaptive Discrete Sliding Mode Control for a Non-minimum Phase ElectroHydraulic Actuator System. International Conference on Robotic, Vision, Signal Processing \& Power Applications. Springer. 2014; 1:3-14.

[13] Seborg DE, Mellichamp DA, Edgar TF, Doyle IIIFJ. Process dynamics and control. John Wiley \& Sons.2010

[14] Emhemed AAA, Mamat R, Hanafi D. Comparison of boiler plant model and real plant responses based on proportional Integral controller. In Sustainable Utilization and Development in Engineering and Technology (STUDENT), 2012 IEEE Conference on. 2012; 1: 117-122.

[15] Clarke DW, Mothadi C, Tu PS. Generalized predictive control Part II. Extensions and interpretations. Automatica. 1987; 23(2): 149-160.

[16] Shridhar R, Cooper DJ. A tuning strategy for unconstrained SISO model predictive control. Industrial \& Engineering Chemistry Research. 1997; 36(3): 729-746.

[17] Ho YK, Yeoh HK, Mjalli FS. Generalized Predictive Control Algorithm with Real-Time Simultaneous Modeling and Tuning. Industrial \& Engineering Chemistry Research. 2014; 53(22): 9411-9426.

[18] Dougherty D, Cooper DJ. Tuning guidelines of a dynamic matrix controller for integrating (non-self-regulating) processes. Industrial \& engineering chemistry research. 2003; 42(8): 1739-1752.

[19] McIntosh AR, Shah SL, Fisher DG. Selection of tuning parameters for adaptive generalized predictive control. In American Control Conference, 1989; 1:1846-1851.

[20] Rossiter JA. Model-based predictive control: a practical approach. CRC press. 2013.

[21] Abu-Ayyad M, Dubay R, Kember GC. SISO extended predictive control—formulation and the basic algorithm. ISA Trans. 2006; 45(1): 9-20.

[22] Abu-Ayyad M, Dubay R. Real-time comparison of a number of predictive controllers. ISA Trans. 2007; 46(3): 411418.

[23] Banerjee P, Shah SL. Tuning guidelines for robust generalized predictive control. In Decision and Control, Proceedings of the 31st IEEE Conference on. 1992; 1: 3233-3234.

[24] Rani KY, Unbehauen H. Study of predictive controller tuning methods. Automatica. 1997; 33(12): 2243-2248.

[25] Emhemed AAA, Mamat R, Faudzi AAM. A New Predictive Control Technique for Force Control of Pneumatic Actuator Plant.The 10th Asian Control Conference (ASCC 2015). 2015; 1:1-6.

\section{BIOGRAPHIES OF AUTHORS}

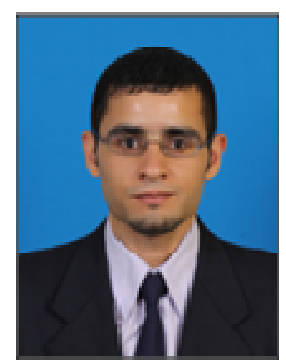

Abdulrahman A.A. Emhemed is a lecturer at College of Electronic Technology-Bani Walid, Libya. Currently he is a PhD Candidate at Control and Mechatronic Engineering Department, Universiti Technologi Malaysia. He has presented many papers at conferences and journals. Mr.Emhemed is a member for many international institutes and engineering associations such as Institute of Electrical and Electronics Engineering (IEEE), Science and Engineering Institute (SCIEI), and the International Association of ENGineers (IAENG). His area of interesting is artificial intelligent, microcontrollers applications, robotics, and process control.

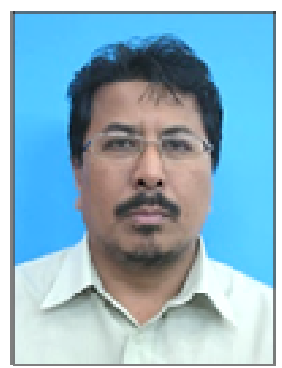

Rosbi Mamat is an associate professor at Department of Control and Mechatronic Engineering at the Faculty of Electrical Engineering, Universiti Teknologi Malaysia. He obtained his $\mathrm{PhD}$ at 1996 in control engineering from University of Sheffield, UK. He has authored and co-authored more many papers in international and local journals and conferences. His research interests include Embedded Systems, Artificial Intelligent Control, Robotics and Mechatronic Systems. 

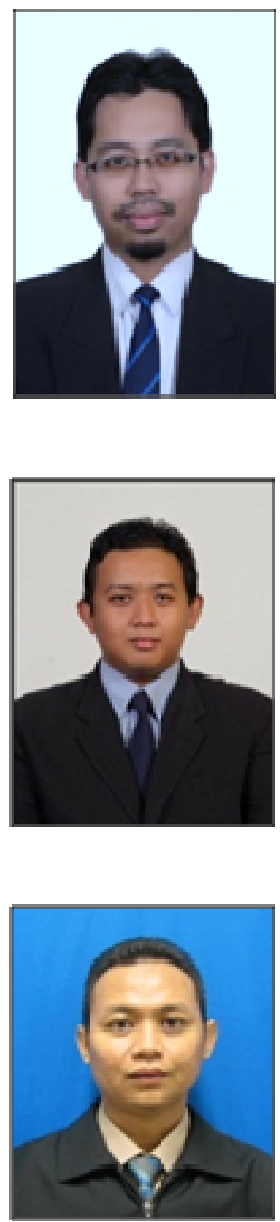

Ahmad `Athif Mohd Faudzi was born in 1982. He received the B. Eng. and the M. Eng. degrees from Universiti Teknologi Malaysia, Malaysia and the Dr. Eng. in System Integration from Okayama University, Japan in 2004, 2006, and 2010 respectively. He is now attached with the Centre for Artificial Intelligence and Robotics (CAIRO), Universiti Teknologi Malaysia as a Senior Lecturer. Dr. Athif is the leader for of the Actuator and Automation Research Group (A2RG). He is mainly engaged in the research fields of pneumatic actuators, soft actuators, robotics automation and their applications.

Mohd Ridzuan Johary is a master student at Universiti Teknologi Malaysia. Mr. Johary is a member of the Actuator and Automation Research Group (A2RG) and supervised by Dr. Ahmad 'Athif Mohd Faudzi. He is main research in the inellegent control integrated with ElectroHydraulic Actuators and their applications.

Khairuddin Osman is a Lecturer at Department of Industrial Electronics, Faculty of Electrical Engineering, Universiti Teknikal Malaysia Melaka. He received his MS.cEng and PhD degrees from Universiti Teknologi Malaysia, Malaysia. He has presented many papers at local and international conferences and journals. His areas of interst include System Identification, as Instrumentation and Automation Systems. 